IJMMS 30:11 (2002) 697-703

PII. S0161171202013017

http://ijmms.hindawi.com

(c) Hindawi Publishing Corp.

\title{
ESTIMATES FOR THE NORMS OF SOLUTIONS OF DELAY DIFFERENCE SYSTEMS
}

\author{
RIGOBERTO MEDINA
}

Received 26 April 2001

We derive explicit stability conditions for delay difference equations in $\mathbb{C}^{n}$ (the set of $n$ complex vectors) and estimates for the size of the solutions are derived. Applications to partial difference equations, which model diffusion and reaction processes, are given.

2000 Mathematics Subject Classification: 39A10.

1. Introduction. Stability of systems of difference equations with delays has been discussed by many authors, for example, see GiL' and Cheng [7], Zhang [11], Elaydi and Zhang [5], Pituk [10], Agarwal [1], and the references therein.

In the stability literature, we can find two major trends: stability using the first approximation Lyapunov method and the direct Lyapunov functional method. For this latter trend, see Zhang and Chen [12], Crisci et al. [4], Lakshmikantham and Trigiante [8], and Agarwal and Wong [2]. By this method many very strong results are obtained. But finding Lyapunov's functionals is usually difficult.

In this paper, we consider a class of perturbed difference equations with several delays and, by means of a Gronwall inequality and the recent estimates for the powers $A^{k}$ of a constant matrix $A$ established in [6, Theorem 1.2.1] we derive explicit stability conditions. Further, we apply our main result to an abstract partial difference equation which models reaction and diffusion processes.

2. Preliminary facts. Let $\mathbb{C}^{n}$ be the set of $n$ complex vectors endowed with a norm $\|\cdot\|$. Let $A$ be an $n \times n$-complex matrix.

Consider in $\mathbb{C}^{n}$ the equation

$$
u_{j+1}=A u_{j}+f_{j}\left(u_{j-\sigma_{1}}, \ldots, u_{j-\sigma_{p}}\right), \quad j=0,1, \ldots,
$$

where $p \geq 1$, and $\sigma_{1}, \sigma_{2}, \ldots, \sigma_{p}$ are nonnegative integers such that $0=\sigma_{1}<\sigma_{2}<\cdots<$ $\sigma_{p}, \sigma_{i} \in \mathbb{Z}^{+}$, and $\mathbb{Z}^{+}$is the set of nonnegative integers, $f_{j}$ maps $\mathbb{C}^{n p}$ into $\mathbb{C}^{n}$, for $j=0,1,2, \ldots$.

We consider (2.1) subject to the initial conditions

$$
u_{j}=\tau_{j}, \quad j=-\sigma_{p},-\sigma_{p}+1, \ldots, 0 .
$$

It is assumed that there are nonnegative sequences $q_{l}(l=1,2,3, \ldots, p)$ such that

$$
\left\|f_{j}\left(u_{j-\sigma_{1}}, \ldots, u_{j-\sigma_{p}}\right)\right\| \leq \sum_{l=1}^{p} q_{l}(j)\left\|u_{j-\sigma_{l}}\right\|^{m}, \quad j=0,1, \ldots
$$

and $m$ is a fixed positive real number. 
Unlike differential equations, discrete equations with the given initial conditions always have a solution.

In order to establish our main result, we use the following discrete Gronwall type inequality.

Theorem 2.1 (see [9]). Assume that

$$
z(k) \leq C+\sum_{i=0}^{k-1} \sum_{j=1}^{p} a_{j}(i) z\left(i-\sigma_{j}\right)^{m}, \quad k \in \mathbb{Z}^{+},
$$

where $m>0,0=\sigma_{1}<\sigma_{2}<\cdots<\sigma_{p}, p \geq 1, C>0, a_{j}(k) \geq 0$ for $j=1,2, \ldots, p$ and $k \in \mathbb{Z}^{+}$, and $z(k) \leq C$ for $k=-\sigma_{p},-\sigma_{p}+1, \ldots, 0$.

(a) If $0<m<1$ and $C \leq 1$, then

$$
z(k) \leq C^{m^{k}} \prod_{i=0}^{k-1}\left[1+\sum_{j=1}^{p} a_{j}(i)\right], \quad k \in \mathbb{Z}^{+} .
$$

(b) If $m=1$, then

$$
z(k) \leq C \prod_{i=0}^{k-1}\left[1+\sum_{j=1}^{p} a_{j}(i)\right], \quad k \in \mathbb{Z}^{+} .
$$

(c) If $m>1$, then

$$
z(k) \leq \frac{C}{\left\{1-(m-1) C^{m-1} \cdot \sum_{i=0}^{k-1} \sum_{j=1}^{p} a_{j}(i)\right\}^{1 /(m-1)}}, \quad k \in \mathbb{Z}^{+},
$$

provided that

$$
1-(m-1) C^{m-1} \sum_{i=0}^{k-1} \sum_{j=1}^{p} a_{j}(i)>0, \quad k \in \mathbb{Z}^{+} .
$$

Let $\lambda_{1}(A), \ldots, \lambda_{n}(A)$ be the eigenvalues of $A$, including their multiplicities. We will make use of the following quantity hereafter (see [6, Chapter 1$]$ ):

$$
g(A)=\left\{N^{2}(A)-\sum_{i=1}^{n}\left|\lambda_{i}(A)\right|^{2}\right\}^{1 / 2},
$$

where $N(A)$ is the Frobenius (Hilbert-Schmidt) norm of $A$, that is, $N^{2}(A)=\operatorname{Trace}\left(A A^{*}\right)$.

There are a number of properties of $g(A)$ which are useful (see [6]). Here, we note that if $A$ is normal, that is, $A A^{*}=A^{*} A$, then $g(A)=0$. If $A=\left(a_{i j}\right)$ is a triangular matrix such that $a_{i j}=0$ for $1 \leq j<i \leq n$, then

$$
g^{2}(A)=\sum_{1 \leq i<j \leq n}\left|a_{i j}\right|^{2} .
$$

To facilitate the description of our main result, we adopt the convention that $0 !=1$, $0^{0}=1$, and that empty sums are zero. Further, the binomial coefficient $C_{j}^{i}$ is given by

$$
C_{j}^{i}=\frac{i !}{j !(i-j) !}, \quad 0 \leq j \leq i .
$$


As normal, but we also adopt the convention that $C_{j}^{i}=0$ when $j<0$ or $j>i$. We define

$$
\gamma_{n, i}= \begin{cases}\sqrt{\frac{C_{i}^{n-1}}{(n-1)^{i}}}, & i=0,1,2, \ldots, n-1, \\ 0, & \text { if } i<0 \text { or } i>n-1 .\end{cases}
$$

Note that

$$
\gamma_{n, i}^{2}=\frac{(n-2)(n-3) \cdots(n-i)}{(n-1)^{i-1} i !} \leq \frac{1}{i !}
$$

Finally, we denote $M=\sup _{m \geq 0} \sum_{k=0}^{n-1} C_{k}^{m} \rho^{m-k}(A) g^{k}(A) \gamma_{n, k}$, where $\rho(A)$ is the spectral radius of $A$.

3. Main result. Now, we are in a position to establish our main result pertaining to the boundedness and convergence to zero of the solutions of (2.1) subject to the initial conditions (2.2).

THEOREM 3.1. Assume that

(i) there are nonnegative sequences $q_{l}(l=1,2, \ldots, p)$ such that

$$
\left\|f_{j}\left(u_{j-\sigma_{1}}, \ldots, u_{j-\sigma_{p}}\right)\right\| \leq \sum_{l=1}^{p} q_{l}(j)\left\|u_{j-\sigma_{l}}\right\|^{m}, \quad j=0,1, \ldots
$$

for $p \geq 1$ and $m$ a fixed positive real number,

(ii) $\sum_{k=0}^{\infty} \sum_{l=1}^{p} q_{l}(k)<\infty$,

(iii) $v_{0}=g(A)<\infty$.

Then,

(a) if $0<m \leq 1$ and $L=M\left\|\tau_{0}\right\| \leq 1$, with

$$
M=\sup _{m \geq 0} \sum_{k=0}^{n-1} C_{k}^{m} \rho^{m-k}(A) g^{k}(A) \gamma_{n, k},
$$

every solution $u_{j}$ of (2.1) and (2.2), such that $\left\|u_{j}\right\| \leq L$ for $j=-\sigma_{p},-\sigma_{p}+1, \ldots, 0$, is bounded, and $\lim _{j \rightarrow \infty}\left\|u_{j}\right\|=0$ whenever $\left\|\tau_{0}\right\|<\delta$, for $\delta>0$ small enough;

(b) if $m>1$ and

$$
\left\|\tau_{0}\right\| \leq\left\{\frac{r}{(m-1) M^{m} \sum_{k=0}^{\infty} \sum_{l=1}^{p} q_{l}(k)}\right\}^{1 /(m-1)},
$$

for an arbitrary real number $r \in(0,1)$, every solution $u_{j}$ of (2.1) and (2.2), satisfying $\left\|u_{j}\right\| \leq L$ for $j=-\sigma_{p},-\sigma_{p}+1, \ldots, 0$, is bounded.

Proof. Note first that by inductive arguments, we can prove that the unique solution $\left\{u_{j}\right\}_{j=-\sigma_{p}}^{\infty}$ of (2.1), subject to given initial values: $u_{0}=\tau_{0}, u_{-1}, \ldots, u_{-\sigma_{p}}$, satisfies

$$
u_{j}=A^{j} \boldsymbol{\tau}_{0}+\sum_{k=0}^{j-1} A^{j-k-1} f_{k}\left(u_{k-\sigma_{1}}, \ldots, u_{k-\sigma_{p}}\right), \quad j=0,1,2, \ldots .
$$


Hence,

$$
\begin{aligned}
\left\|u_{j}\right\| & \leq\left\|A^{j}\left|\left\|\left|\tau_{0}\left\|+\sum_{k=0}^{j-1}\right\| A^{j-k-1}\right|\right\|\right| f_{k}\left(u_{k-\sigma_{1}}, \ldots, u_{k-\sigma_{p}}\right)\right\| \\
& \leq\left\|A^{j}\right\|\left\|\tau_{0}\right\|+\sum_{k=0}^{j-1}\left\|A^{j-k-1}\right\| \sum_{l=1}^{p} q_{l}(k)\left\|u_{k-\sigma_{l}}\right\|^{m} .
\end{aligned}
$$

Denote $\Gamma=\sup _{j \geq 0}\left\|A^{j}\right\|$. Thus, we have

$$
\begin{aligned}
\left\|u_{j}\right\| & \leq \Gamma|| \tau_{0}\left\|+\sum_{k=0}^{j-1} \sum_{l=1}^{p} \sup _{j \geq k \geq 0}\right\| A^{j-k-1}\left\|q_{l}(k)\right\| u_{k-\sigma_{l}} \|^{m} \\
& \leq \Gamma\left\|\tau_{0}\right\|+\Gamma \sum_{k=0}^{j-1} \sum_{l=1}^{p} q_{l}(k)\left\|u_{k-\sigma_{l}}\right\|^{m} .
\end{aligned}
$$

We now recall from [6] that

$$
\left\|A^{j}\right\| \leq \sum_{k=0}^{\min \{j, n-1\}} C_{k}^{j} \rho^{j-k}(A) g^{k}(A) \gamma_{n, k}
$$

which implies that

$$
\Gamma=\sup _{j \geq 0}\left\|A^{j}\right\| \leq \sup _{j \geq 0} \sum_{k=0}^{n-1} C_{k}^{j} \rho^{j-k}(A) g^{k}(A) \gamma_{n, k}=M .
$$

Thus, it follows that

$$
\left\|u_{j}\right\| \leq M\left\|\tau_{0}\right\|+M \sum_{k=0}^{j-1} \sum_{l=1}^{p} q_{l}(k)\left\|u_{k-\sigma_{l}}\right\|^{m} .
$$

Put $v(j)=\left\|u_{j}\right\|$ for $j=0,1,2, \ldots$, hence

$$
v(j) \leq L+M \sum_{k=0}^{j-1} \sum_{l=1}^{p} q_{l}(k) v\left(k-\sigma_{l}\right)^{m}
$$

where $L=M\left\|\tau_{0}\right\|$ and $v(j) \leq L$ for $j=-\sigma_{p},-\sigma_{p}+1, \ldots, 0$.

CASE 1. If $0<m \leq 1$ and $L \leq 1$, then by (2.4) and Theorem 2.1(a) we have

$$
v(j) \leq L^{m^{j}} \prod_{k=0}^{j-1}\left[1+\sum_{l=1}^{p} q_{l}(k)\right] \leq L^{m^{j}} \exp \left(M \sum_{k=0}^{\infty} \sum_{l=1}^{p} q_{l}(k)\right) .
$$

Thus, establishing that the solution $u_{j}$ is bounded for $j=-\sigma_{p},-\sigma_{p}+1, \ldots, 0$, and $\lim _{j \rightarrow \infty}\left\|u_{j}\right\|=0$ whenever $\left\|u_{0}\right\|<\delta$, for $\delta>0$ small enough.

CASE 2. If $m>1$, then proceeding in a similar way to Case 1 , we arrive at inequality (3.10). 
Hence, by Theorem 2.1(b), it follows that

$$
v(j) \leq \frac{L}{\left\{1-(m-1) L^{m-1} \sum_{k=0}^{j-1} \sum_{l=1}^{p} q_{l}(k)\right\}^{1 /(m-1)}}
$$

provided that

$$
1-(m-1) L^{m-1} \sum_{k=0}^{j-1} \sum_{l=1}^{p} q_{l}(k)>0 .
$$

Let $r \in(0,1)$ be an arbitrary real number. We prove that condition (3.13) holds for all $\tau$ satisfying

$$
\|\boldsymbol{\tau}\| \leq\left\{\frac{r}{(m-1) M^{m} \gamma}\right\}^{1 /(m-1)}=: R,
$$

where

$$
\gamma=\sum_{k=0}^{\infty} \sum_{l=1}^{p} q_{l}(k)<\infty .
$$

Indeed, for all such a $\tau_{0}$, we have

$$
\begin{aligned}
& (m-1) M^{m-1}\left\|\tau_{0}\right\|^{m-1} \sum_{k=0}^{j-1} \sum_{l=1}^{p} q_{l}(k) \\
& \leq(m-1) M^{m-1}\left\|\tau_{0}\right\|^{m-1} \sum_{k=0}^{\infty} \sum_{l=1}^{p} q_{l}(k) \leq r .
\end{aligned}
$$

Thus,

$$
1-(m-1) M^{m} L^{m-1} \sum_{k=0}^{j-1} \sum_{l=1}^{p} q_{l}(k) \geq 1-r>0 .
$$

Consequently, for all $\tau$ such that $\left\|\tau_{0}\right\| \leq R$, we have

$$
\begin{aligned}
\left\|u_{j}\right\| & \leq \frac{M\left\|\tau_{0}\right\|}{\left\{1-(m-1) L^{m-1} \sum_{k=0}^{j-1} \sum_{l=1}^{p} M q_{l}(k)\right\}^{1 /(m-1)}} \\
& \leq \frac{M}{(1-r)^{1 /(m-1)}}\left\|\tau_{0}\right\|, \quad j=0,1,2, \ldots
\end{aligned}
$$

Therefore, we have the boundedness of the solution $u_{j}$ of (2.1), subject to the initial conditions (2.2), concluding the proof.

4. Application. In this section, we illustrate our main result by considering an abstract partial difference equation, which models reaction and diffusion processes (see Cheng and Medina [3]). 
Consider a simple three-level discrete reaction-diffusion equation of the form

$$
u_{i, j+1}=a u_{i-1, j}+b u_{i, j}+c u_{i+1, j}+\sum_{i=1}^{p} q_{l}(j) u_{i, j-\sigma_{l}},
$$

defined on the set

$$
\Omega=\{(i, j) \mid i=0,1, \ldots, n+1, j=0,1, \ldots\},
$$

where $q_{l}(l=1,2, \ldots, p)$ are nonnegative real sequences; $u_{i, j}$ are complex sequences, $p \geq 1 ; a, b, c$ are real numbers; and $0=\sigma_{1}<\sigma_{2}<\cdots<\sigma_{p}, \sigma_{i} \in \mathbb{Z}^{+}$.

For the sake of simplicity, Dirichlet boundary conditions of the form

$$
u_{0, j}=0=u_{n+1, j}, \quad j=0,1, \ldots
$$

will be imposed.

Given an arbitrary set of initial values $u_{i, j},-\sigma_{p} \leq j \leq 0,1 \leq i \leq n$, namely

$$
u_{i, j}=\tau_{i, j}, \quad-\sigma_{p} \leq j \leq 0,1 \leq i \leq n .
$$

We can successively calculate $u_{1,1}, u_{2,1}, \ldots, u_{n, 1} ; u_{1,2}, \ldots, u_{n, 2} ; \ldots$, according to (4.1) in a unique manner. Such a double sequence: $u=\left\{u_{i, j} \mid i=0,1, \ldots, n+1, j=-\sigma_{p},-\sigma_{p}+\right.$ $1, \ldots\}$ is called a solution of (4.1) subject to conditions (4.3) and (4.4). An existence and uniqueness theorem for (4.1) can thus be formulated and proved in a straightforward manner.

By designating $\operatorname{col}\left(u_{1, j}, u_{2, j}, \ldots, u_{n, j}\right)$ as the $C^{n}$-vector $u_{j}$, we see that a solution of (4.1), (4.3), and (4.4) can also be regarded as a vector sequence $\left\{u_{j}\right\}_{j=-\sigma_{p}}^{\infty}$. Furthermore, such a sequence satisfies the delay vector recurrence relation

$$
u_{j+1}=A u_{j}+\sum_{i=1}^{p} q_{l}(j) u_{j-\sigma_{l}},
$$

subject to the initial conditions

$$
u_{j}=\tau_{j}, \quad j=-\sigma_{p},-\sigma_{p}+1, \ldots, 0,
$$

where $\tau_{j}=\operatorname{col}\left(\tau_{1, j}, \tau_{2, j}, \ldots, \tau_{n, j}\right)$, and

$$
A=\left[\begin{array}{cccccc}
b & c & 0 & 0 & \cdots & 0 \\
a & b & c & 0 & \cdots & 0 \\
0 & a & b & c & \cdots & 0 \\
0 & 0 & a & b & \cdots & 0 \\
\vdots & \vdots & \vdots & \vdots & \vdots & \vdots \\
0 & 0 & \cdots & \cdots & a & b
\end{array}\right],
$$

In particular, (4.5) is of the form (2.1), where

$$
f_{j}\left(u_{j-\sigma_{1}}, \ldots, u_{j-\sigma_{p}}\right)=\sum_{i=1}^{p} q_{l}(j) u_{i, j-\sigma_{l}}, \quad j=0,1,2, \ldots .
$$


THEOREM 4.1. Let conditions (ii) and (iii) of Theorem 3.1 hold. Further, assume that

$$
\left\|f_{j}\left(u_{j-\sigma_{1}}, \ldots, u_{j-\sigma_{p}}\right)\right\| \leq \sum_{l=1}^{p} q_{l}(j)\left\|u_{j-\sigma_{l}}\right\|, \quad j=0,1, \ldots
$$

Then, if $L=M\left\|\tau_{0}\right\| \leq 1$, with $M=\sup _{j \geq 0} \sum_{k=0}^{n-1} C_{k}^{j} \rho^{j-k}(A) g^{k}(A) \gamma_{n, k}$, every solution $u_{j}$ of (4.5) and (4.6), such that $\left\|u_{j}\right\| \leq L$, for $j=-\sigma_{p},-\sigma_{p}+1, \ldots, 0$, is bounded, and $\lim _{j \rightarrow \infty}\left\|u_{j}\right\|=0$ whenever $\left\|\tau_{0}\right\|<\delta$, for $\delta>0$ small enough.

Proof. The proof is a direct consequence of Theorem 3.1.

AcKnowledgment. This research was supported by Fondecyt under Grant No. 1000023.

\section{REFERENCES}

[1] R. P. Agarwal, Difference Equations and Inequalities, Marcel Dekker, New York, 1992.

[2] R. P. Agarwal and P. J. Wong, Advanced Topics in Difference Equations, Mathematics and Its Applications, vol. 404, Kluwer Academic Publishers, Dordrecht, 1997.

[3] S. S. Cheng and R. Medina, Growth conditions for a discrete heat equation with delayed control, Dynam. Systems Appl. 8 (1999), no. 3-4, 361-367.

[4] M. R. Crisci, V. B. Kolmanovskii, E. Russo, and A. Vecchio, Stability of difference Volterra equations: direct Lyapunov method and numerical procedure, Comput. Math. Appl. 36 (1998), no. 10-12, 77-97.

[5] S. Elaydi and S. Zhang, Stability and periodicity of difference equations with finite delay, Funkcial. Ekvac. 37 (1994), no. 3, 401-413.

[6] M. I. GiL', Norm Estimations for Operator-Valued Functions and Applications, Monographs and Textbooks in Pure and Applied Mathematics, vol. 192, Marcel Dekker, New York, 1995.

[7] M. I. GiL' and S. S. Cheng, Stability of a time discrete perturbed dynamical system with delay, Discrete Dynamics in Nature and Society 3 (1999), 57-63.

[8] V. Lakshmikantham and D. Trigiante, Theory of Difference Equations, Academic Press, Massachusetts, 1988.

[9] V. N. Phat and J. Y. Park, On the Gronwall inequality and asymptotic stability of nonlinear discrete systems with multiple delays, Dynam. Systems Appl. 9 (2000), no. 2, 309321.

[10] M. Pituk, Convergence and uniform stability in a nonlinear delay difference system, Math. Comput. Modelling 22 (1995), no. 2, 51-57.

[11] S. Zhang, Estimate of total stability of delay difference systems, Comput. Math. Appl. 37 (1999), no. 9, 31-38.

[12] S. Zhang and M. Chen, A new Razumikhin theorem for delay difference equations, Comput. Math. Appl. 36 (1998), no. 10-12, 405-412.

Rigoberto Medina: Departmento de Ciencias Exactas, Universidad de Los Lagos, CASilla 933, Osorno, Chile 


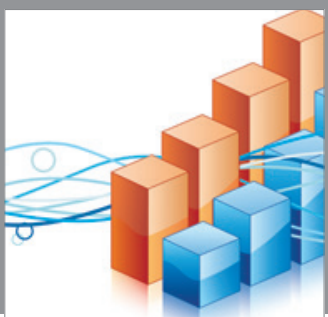

Advances in

Operations Research

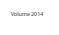

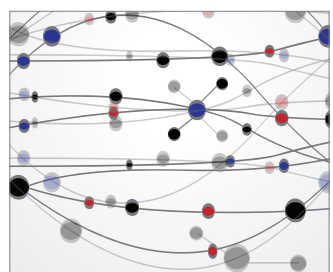

\section{The Scientific} World Journal
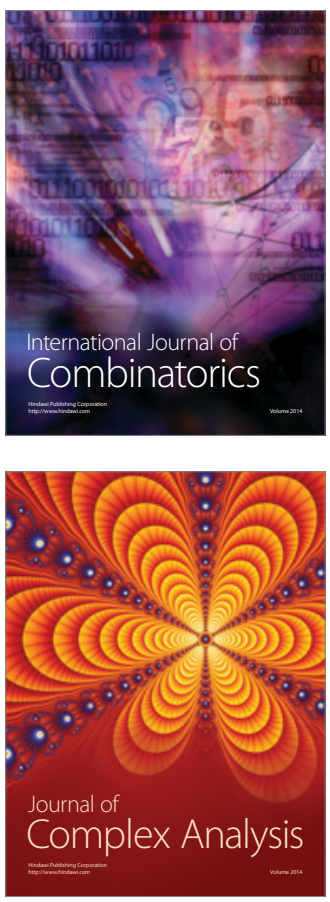

International Journal of

Mathematics and

Mathematical

Sciences
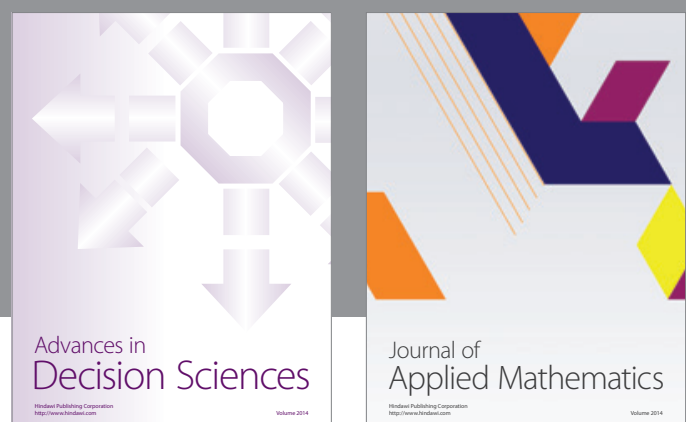

Journal of

Applied Mathematics
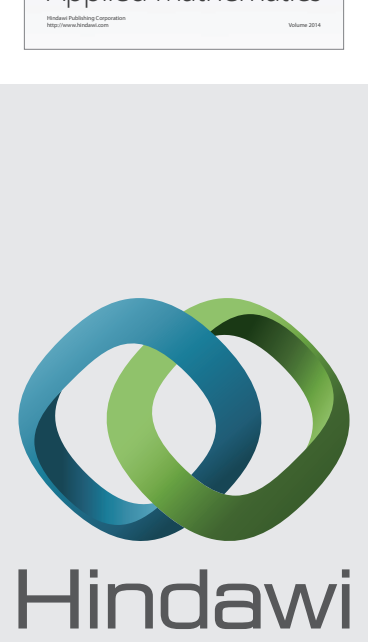

Submit your manuscripts at http://www.hindawi.com
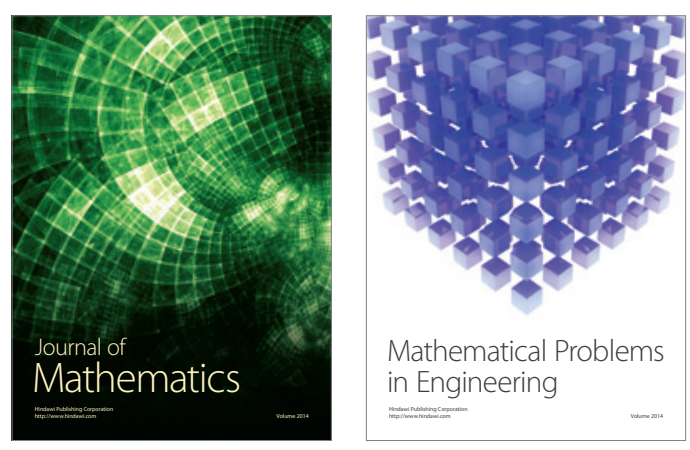

Mathematical Problems in Engineering
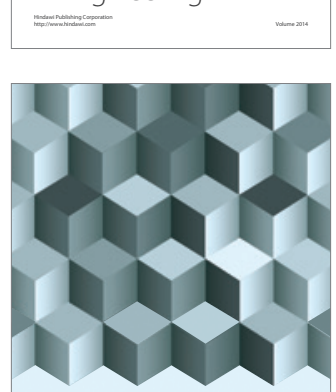

Journal of

Function Spaces
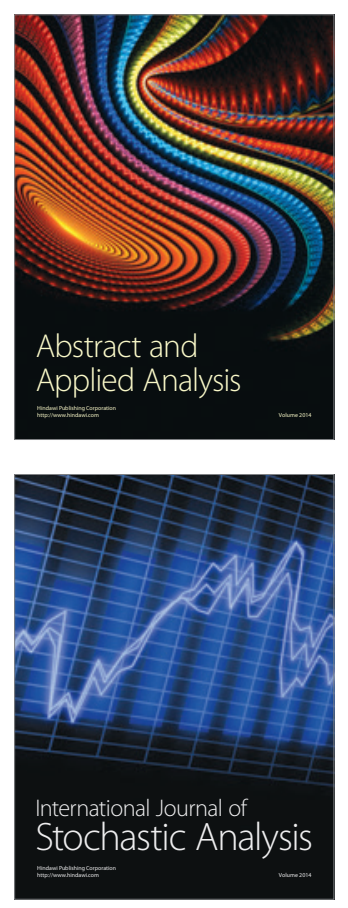

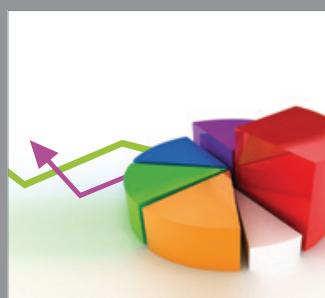

ournal of

Probability and Statistics

Promensencen
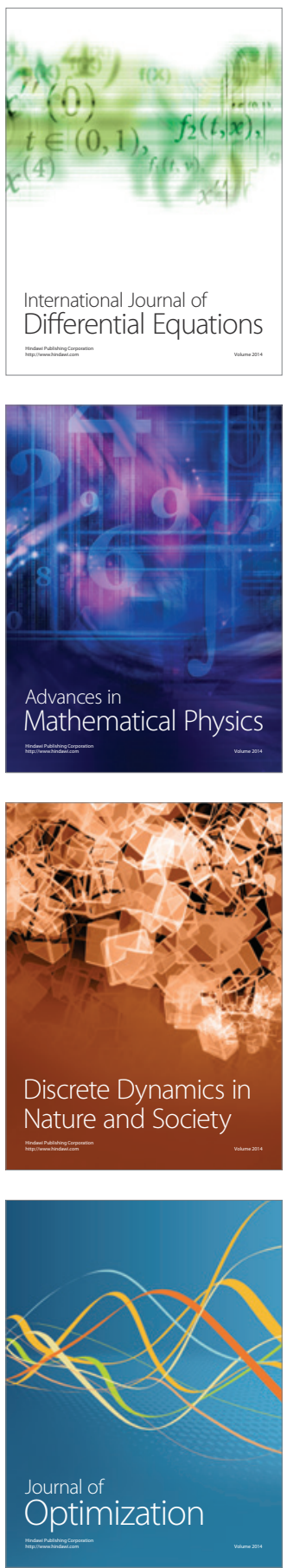